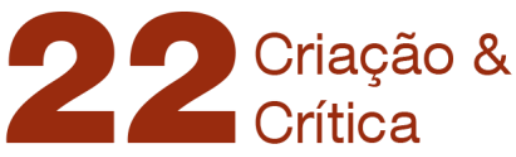

\section{LA VALISE DE L'ÉCRIVAIN}

\section{Variations sur le Thème de la Lecture dans L'énigme du Retour, de Dany Laferrière}

\author{
Henrique Provinzano Amaral ${ }^{12}$
}

RESUME : Cet article est consacré au roman L'Énigme du retour, du célèbre écrivain haïtien Dany Laferrière. L'image de la valise, très présente dans le récit, figure à la fois l'idée d'héritage et ce qui permet de rassembler des œuvres fondamentales. Ainsi, le voyage littéraire du narrateur part des classiques universels jusqu'à la littérature caribéenne la plus contemporaine. Finalement, grâce au concept de " posture » (Jérôme Meizoz, 2007), ce travail vise à interpréter les diverses variations du thème de la lecture dans le roman.

MOTS-CLES : Dany Laferrière ; lecture littéraire ; littérature haïtienne ; posture.

\section{The Writer's Valise: Variations on the Theme of Reading IN DANY LAFERRIĖRE'S L'ÉNIGME dU RETOUR}

ABSTRACT: This article concerns the prize winner Caribbean writer Dany Laferrière and his novel L'Énigme du retour. Showing up throughout the novel's narrative, the valise as an image represents at the same time the idea of heritage and a repository of fundamental literary works. Therefore, the narrator's patch goes from universal classics to the most contemporary Caribbean Literature. Finally, thanks to a concept such as "posture" (Jérôme Meizoz, 2007), this work intends to explore in this novel the multiple variations on the theme of reading.

KEYWORDS: Dany Laferrière ; literary lecture ; haitian literature ; posture.

Je passerais bien ici le reste de mon temps

à bavarder de tout et de rien avec des gens qui n'ont jamais ouvert un livre de leur vie. Et viendra tôt ou tard l'instant précieux où je confondrai les romans que j'ai lus avec ceux que j'ai écrits.

Dany Laferrière, L'énigme du retour

1 Mestrando do PPG LETRA (Letras Estrangeiras e Tradução) - FFLCH/USP. E-mail: henrique.provinzano.amaral@usp.br

20 presente trabalho foi realizado com apoio da Coordenação de Aperfeiçoamento de Pessoal de Nível Superior - Brasil (CAPES) - Código de financiamento 001. This study was financed in part by the Coordenação de Aperfeiçoamento de Pessoal de Nível Superior - Brasil (CAPES) - Finance Code 001. 


\section{Lents préparatifs de départ ou la valise de l'écrivain-lecteur}

Le roman publié par l'écrivain haïtien Dany Laferrière en 2009, intitulé L'Énigme du retour, présente comme épigraphe les premiers mots du très connu Cahier d'un retour au pays natal, du Martiniquais Aimé Césaire - "Au bout du petit matin... ». En lisant ce poème, on comprend que ces mots-là se répètent comme une espèce de leitmotiv poétique capable d'introduire différentes parties du texte. Placée donc à la tête du roman de Laferrière, en guise de citation ou de confusion avouée entre les livres qu'il a lus et ceux qu'il a écrits, la célèbre phrase de Césaire devient aussi un leitmotiv narratif, du moins sur deux plans principaux. Le premier est le plan plutôt temporel de l'organisation de l'intrigue : on lit au début du premier chapitre (« Le coup de fil ») de la première partie du livre, nommée "Lents préparatifs de départ», que "la nouvelle coupe la nuit en deux » : le narrateur apprend, au bout du petit matin, que "[son] père vient de mourir » (LAFERRIĖRE, 2009, p. 13). C'est cette triste nouvelle qui entraîne l'écrivain, à la première page de son récit, dans un voyage de retour à sa mémoire personnelle, bien qu'à son pays natal.

Ainsi, au deuxième plan, au tout début de la narration, on peut se rendre compte qu'il y aura un mouvement de retour, c'est-à-dire, de (re)lecture du passé et du présent, dont un important point de repère est le poème de Césaire, qui présente le mot retour dans son titre même. II faut avoir en tête que le Cahier, paru pour la première fois en 1939, est généralement considéré comme le chef-d'œuvre de son auteur, ainsi que comme le texte fondateur du mouvement poétique de la Négritude, et même comme l'une des œuvres principales de la littérature francophone ${ }^{3}$. II ne faut pas oublier non plus le rôle important joué dans ce poème par l'image d'Haïti, fréquemment présente à travers les figures de Toussaint Louverture et de sa révolution des esclaves: "[...] Haïti où la négritude se mit debout pour la première fois et dit qu'elle croyait à son humanité [...] » (CÉSAIRE, 1983, p. 24).

De nombreuses analyses ont déjà été faites sur les rapports entre le poème de Césaire et l'histoire haïtienne, aussi bien qu'entre celle-ci et l'émergence de la Négritude ${ }^{4}$. Néanmoins, mon analyse du roman de Laferrière ne se consacre pas exactement à cette place occupée par le Cahier dans l'histoire de la littérature francophone ou antillaise, mais à sa position en tant qu'intertexte privilégié de L'Énigme du retour. II faut remarquer à ce propos que les citations et les allusions au poème ne sont pas limitées à la seule position d'épigraphe, mais éparpillées partout dans le roman. Dans l'article « De l'obstacle à la transparence : L'Énigme du retour de Dany Laferrière ou la réponse limpide de l'écriture vibrante », le critique Anthony Soron établit un parallèle très intéressant entre la figure du poète Césaire et celle du père décédé, le premier agissant comme une présence plus tangible par rapport à l'absence de contours du deuxième :

3 Lilian P. de Almeida, spécialiste brésilienne de l'œuvre de Césaire et traductrice du poème en question, le décrit ainsi dans la postface de sa traduction: "Le Cahier d'un retour au pays natal, véritable discours serpent, est le poème le plus connu du groupe de textes de ce que l'on appelle aujourd'hui la francophonie et fonde poétiquement la négritude » (ALMEIDA, 2012, p. 93, traduction du portugais faite par moi). L'édition du Cahier d'un retour au pays natal qu'on cite en français est celle des éditions Présence Africaine, 1983.

4 Cf., par exemple, DEPESTRE, René. Bonjour et adieu à la négritude. Paris : Éditions Robert Laffont, 1980. 


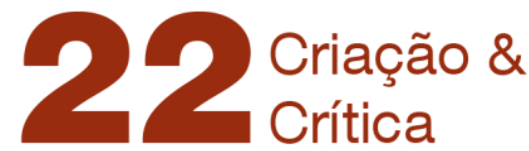

L'imaginaire de l'écrivain semble dépositaire de deux photos patriciales, celle de son père, Windsor Laferrière, et celle d'«Un poète nommé Césaire " pour reprendre le titre même d'un chapitre du roman. Tandis qu'il éprouve les pires difficultés à remettre en mouvement les traits du visage de Windsor, ceux du poète antillais s'animent sans effort dans son imagination [...]. (SORON, 2013, p. 156-157)

De ce point de vue, située comme le double de l'image manquante du père, celle d'Aimé Césaire apparaît sans cesse, pas seulement en tant que poète, mais aussi comme l'homme enragé et engagé qu'il fut : " II y a une photo de Césaire / où on le voit assis sur un banc. / La mer derrière lui. / [...] Son sourire fané / et ses grands yeux si doux / ne laissent pas deviner cette rage / qui le change, sous nos yeux, / en un tronc d'arbre calciné » (LAFERRIĖRE apud SORON, 2013, p. 157). Au moment des lents préparatifs de départ, c'est plutôt la photo de cet écrivain que le narrateur porte en lui.

En effet, dans cette première partie du roman, Dany Laferrière entreprend une série de voyages : tout au début, après cet appel téléphonique où on lui apprend le décès de son père, il prend la route en voiture et "sans destination " vers le nord du Canada. Quoiqu'il ne semble pas assez justifié en termes narratifs, ce premier essai de déplacement fonctionne déjà comme un éloignement partiel du paysage habituel de Montréal, ville où demeure l'écrivain. En revanche, il est évident que le chemin plein de neige, les " petits villages engourdis / le long d'un fleuve gelé », bref ce " règne de I'hiver " constituent l'exact contraire de ce qu'il retrouve plus tard, dans la chaleur de son Haïti natal. L'auteur lui-même est bien « conscient d'être dans un monde/ à l'opposé du [sien] $)$.

Parmi les quelques apparitions constatées dans cette route gelée, dont celle d'une " petite fille aux cheveux noirs / et à la robe jaune fièvre ${ }^{5}$, il faut remarquer l'étrange figure d'un autre écrivain : " Je sais qu'au bout de cette route / un barbu plein de fureurs et de douceurs, / au milieu d'une meute de chiens, / tente d'écrire le grand roman américain » (2009, p. 16).

II est presque totalement vain d'essayer de deviner qui est ce « barbu plein de fureurs et de douceurs » et quel est le livre qu'il tente d'écrire. Ce que l'on doit retenir c'est plutôt cette représentation presque mythologique d'un auteur qui vit en ermite, au milieu de la glace et d'une meute de chiens, en écrivant " le grand roman américain ». On sait que Dany Laferrière lui-même donne le nom d'Autobiographie américaine à une partie considérable de son $œ u v r{ }^{6}$. Ainsi, peut-on s'interroger: y aurait-il une sorte de miroitement entre les deux écrivains, celui qui écrit et l'autre qui est décrit ? Ou encore, d'où pourrait émaner ce grand roman américain : de la « glace du Nord » ou du «feu du

\footnotetext{
5 Quoiqu'il ne soit pas évident, on peut rapprocher cette petite fille à la robe jaune de la figure de Vava, le premier amour haïtien de l'auteur, image obsédante qui revient dans de nombreux livres, dont quelques-uns pour les enfants (Je suis fou de Vava, Le baiser mauve de Vava).

6 Le corpus formé par Comment faire l'amour avec un nègre sans se fatiguer, publié en 1985, et les neuf romans qui le suivent, a été appelé par Laferrière, à plusieurs reprises, " une autobiographie américaine ».
} 


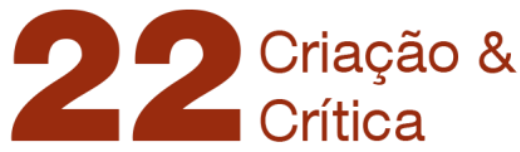

Sud "? Ce sont des questions relevant d'une mythologie personnelle appliquée à la littérature, c'est-à-dire de la façon dont Laferrière se met en relation avec d'autres écrivains, incluant également sa position en tant qu'auteur. Sans chercher à répondre immédiatement à ces questions, il convient de noter qu'après ce court voyage, le narrateur revient chez lui.

En fait, le rythme de la première partie est lent, marqué par un va-et-vient qui ressemble plutôt à une gestation de la deuxième et plus longue partie, "Le retour ". Rentré à Montréal, le narrateur doit faire sa valise, dans laquelle il jette " deux ou trois jeans, trois chemises, deux paires de chaussures, quelques sous-vêtements, un tube de dentifrice, deux brosses à dents, une boîte d'aspirine et [son] passeport ". Malgré son propre discours ( « Je repartirai avec une petite valise. / Comme celle que j'avais en arrivant ici. / Presque vide. / Pas un seul livre. / Même pas les miens ॥), on sait qu'au départ, aussi bien qu'au retour, l'auteur emportait avec lui un exemplaire du Cahier d'un retour au pays natal:

Je reviens à ma première valise oubliée dans une de ces chambres étroites et poussiéreuses de la ville. Heureusement que j'avais pu récupérer les seules choses qui valaient la peine. Une lettre de ma mère où elle m'explique, par le menu, comment vivre dans un pays qu'elle n'a jamais visité, et cet exemplaire fripé du Cahier d'un retour au pays natal du poète martiniquais Aimé Césaire. Je les garde toujours sur moi. (LAFERRIĖRE, 2009, p. 56)

Par le biais de la répétition, on peut remarquer que le contenu de la valise n'est pas une question anodine. Répété tout au long de l'ouvrage, il fonctionne comme le signe même de l'idée d'héritage : d'après cet extrait, "les seules choses qui valaient la peine " dans la première valise, celle de l'arrivée, étaient la lettre de la mère et « cet exemplaire fripé » du Cahier de Césaire. Deux objets qui n'ont comme valeur que leur existence en tant que discours, le premier apprenant «comment vivre dans un pays qu'elle [la mère] n'a jamais visité ", le second fonctionnant peut-être comme un guide de retour au pays natal. Ainsi, le rapport entre ces deux écrits est de complémentarité, comme il pourrait l'être entre les figures maternelle et paternelle.

À propos du thème de la lecture, Véronique Bonnet écrit :

Le lecteur-écrivain qu'est Laferrière nous convie aussi au sein de sa propre bibliothèque, vaste, érudite, vénérée, patiemment édifiée, dans laquelle l'éclectisme affiché fait la part belle à la présence pointée des classiques: Baldwin et Césaire, Borges et Proust [...] s'y côtoient et rencontrent les Anciens, Sénèque, Homère, Érasme, pour ne citer que quelques-uns, lors de joyeuses et néanmoins savantes bacchanales littéraires, ponctuées de portraits d'écrivains, étayées par des fragments de leurs œuvres, des commentaires, des réécritures, des dialogues. (BONNET, 2016, p. 256). 


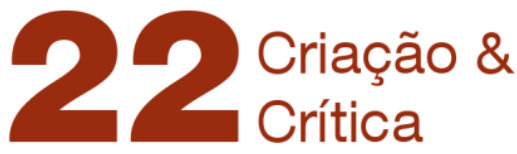

En effet, l'article de Véronique Bonnet intitulé « Dany Laferrière : le lecteur dans sa baignoire ou l'art de bien lire » fournit des bases théoriques et critiques essentielles pour ma lecture de L'Énigme du retour. En suivant cette trace on peut observer que, dans ce roman, le Cahier d'un retour au pays natal constitue un intertexte si évident et central (visible déjà dans les titres), qu'il n'a même pas besoin d'être compté comme tel, conformément à ce qui a déjà été constaté au sujet du contenu des valises. Cependant, les autres intertextes - ceux que l'on pourrait reconnaître comme étant moins centraux dans l'œuvre -, sont explicités plus clairement :

J'attends d'être dans un sanatorium pour plonger dans Les Buddenbrook du sévère Thomas Mann ou suivre à la trace Le Guépard de Giuseppe Tomasi di Lampedusa. Pourquoi gardons-nous des livres que nous ne lirons jamais ? Pour Le Guépard le nom de l'auteur à lui seul valait la dépense. J'ai oublié ce qui m'a empêché de lire le roman de Thomas Mann. (LAFERRIÈRE, 2009, p. 43)

Dans ce chapitre («Le temps des livres »), l'auteur met en scène l'une de ses multiples réflexions sur la lecture, cette fois-ci en insistant sur le contraste entre ses livres d'avant l'exil, qui étaient peu nombreux et qu'il lisait à plusieurs reprises, et sa bibliothèque d'écrivain bien établi au Canada: "Aujourd'hui je constate qu'il me reste à lire / une bonne moitié de ma bibliothèque" (2009, p. 43). En fait, la digression sur Les Buddenbrook, "du sévère Thomas Mann » et Le Guépard, de Lampedusa, se révèle quasi totalement futile. Effectivement, ces œuvres ne sont là que comme des livres qui n'ont pas été lus : pour Le Guépard, "le nom de l'auteur à lui seul valait la dépense "; pour Les Buddenbrook, l'auteur ne se rappelle même pas la raison de cette non lecture. En outre, la volonté reconnue de s'abstenir de vie sociale (« J'attends d'être dans un sanatorium ") pour se consacrer à plein temps à la lecture rejoint ce que Laferrière luimême appelle, dans Journal d'un écrivain en pyjama, l'attitude de "l'écrivain-lecteur ». Dans le petit extrait sous le titre « Lire, lire, lire », il écrit :

Il y a enfin ce que j'appelle l'écrivain-lecteur dont Borges est le plus illustre. II pratique un art très difficile qu'on pourrait appeler «l'érudition merveilleuse ». II plonge dans la bibliothèque comme dans une mer pour ramener à la surface des pépites qui s'appellent Dante, Coleridge, Quevedo, Cervantès, Whitman, Wilde, Chesterton (l'un de ses favoris), De Quincey, Kafka, Keats, Layamon, et naturellement Shakespeare. [...] Borges raconte qu'un jour il est entré dans la bibliothèque de son père et n'en est plus jamais ressorti. Ses livres fourmillent d'écrivains, de réflexions sur les livres qui s'empilent sur sa table de chevet. (LAFERRIĖRE, 2013, p. 84)

Dans cet extrait, l'auteur évoque Borges, le plus illustre des écrivains-lecteurs, connaisseur en l'art de l'érudition merveilleuse, pour caractériser une attitude qui pourrait 
bien être la sienne : celle qui consiste à plonger « dans la bibliothèque comme dans une mer pour ramener à la surface » des livres classiques, tels que Dante et Cervantès. La dernière phrase s'appliquerait ainsi, sans besoin de corrections, à la méthode de l'écrivain Dany Laferrière, dont « les livres fourmillent d'écrivains, de réflexions sur les livres qui s'empilent sur sa table de chevet ». L'effet de proximité est si grand qu'il peut déclencher le souvenir suivant :

Je me souviens qu'un jour, j'ai voulu en avoir le cœur net à leur sujet [des classiques]. C'était au début de l'été. Je suis allé acheter une bonne vingtaine de bouquins d'écrivains de l'Antiquité - ils coûtent moins cher que les autres [...] et je les ai ramenés à la maison comme une meute de chiens errants. J'avais faim de nourriture solide. Je suis entré dans la salle de bains pour ne plus sortir de l'été. (LAFERRIĖRE, 2013, p. 85)

La formule finale - « Je suis entré dans la salle de bains pour ne plus sortir de l'été ॥ - ressemble directement, même si c'est à une moindre échelle, à celle dédiée à l'écrivain argentin - «Borges raconte qu'un jour il est entré dans la bibliothèque de son père et n'en est plus jamais ressorti ». En plus, à travers les images aquatiques, l'attitude du lecteur Laferrière reprend parfaitement celle de plonger dans les lectures classiques, reconnue chez Borges. La même question s'applique à tous les deux : « Est-il un lecteur qui écrit ou un écrivain qui lit ? "(LAFERRIĖRE, 2013, p. 84). Bien évidemment, la bonne réponse n'est pas l'un ou l'autre, mais la combinaison des deux, comme peut indiquer ce trait d'union présent dans l'expression écrivain-lecteur.

II faut encore noter que le fait qu'il s'agisse de lectures supposément classiques n'est pas non plus anodin. La liste présentée - Dante, Coleridge, Quevedo, Cervantès, Whitman etc. - passe à côté d'un critère lié à l'idée de littérature nationale, ou même du choix d'une langue préférée, pour rejoindre ce que l'on appelle habituellement les classiques universe/s. C'est un processus d'appropriation qui rappelle ce que Laferrière dit dans son volume d'entretiens intitulé J'écris comme je vis, en répondant à une question sur l'éducation reçue en Haïti :

Tout cela pour dire que, quand nous [les Haïtiens] étudions Molière, Racine ou Voltaire, nous étudions simplement de grands écrivains et non des Français. Pour les Haïtiens, Corneille, avec ses accents de fierté, ses élans de courage, ses éclats de jeunesse et de noblesse, sa fièvre, est fondamentalement haïtien. Aucun doute là-dessus. Ce nationalisme constitue autant notre force que notre faiblesse. (LAFERRIĖRE, 2000, p. 26)

Le nationalisme attribué par Laferrière au peuple haïtien consiste justement en son contraire, c'est-à-dire en ce qu'il rend national tout ce qui vient d'ailleurs, dans un exercice qui fait de Molière, Racine et Voltaire «simplement de grands écrivains et non des Français ». Dans le cas spécifique de Corneille, ses caractéristiques - fierté, courage, 
jeunesse, noblesse, fièvre - deviennent fondamentalement haïtiennes, dans la mesure où elles se mettent en parallèle avec le caractère supposé de ce peuple. À part une improbable vérification anthropologique de ces affirmations, il convient de rapprocher ce que l'auteur décrit comme étant le comportement général des Haïtiens et sa procédure elle-même. Ce bref examen peut être éclairant en ce qui concerne la relation que l'écrivain entretient avec sa culture d'origine, aussi bien qu'avec la littérature nationale haïtienne. Dans l'ouvrage collectif Pour une littérature-monde, Dany Laferrière écrit :

J'ai perdu trop de temps à commenter le fait que j'écris en français. Et à débattre du fait que ce ne soit pas ma langue maternelle. Finalement, tout cela me paraît aujourd'hui assez théorique, et même un brin ridicule. Cette langue française s'est infiltrée dans mes neurones, et son chant rythme mon sang. Je pourrais reconnaître sa cadence dans une ruelle obscure de Bornéo. Autrefois, je n'aurais jamais admis une telle vérité par peur de découvrir en moi le colonisé. Mais le colonisé, je peux le dire, c'est celui qui ne se voit ni ne s'entend. II se nourrit de mensonges. Sa vie est une fiction. À plus de cinquante ans, il est temps que je mette un peu d'ordre dans ce grenier rempli d'idéologies ringardes qu'est mon esprit. J'écris et je lis en français partout dans le monde. C'est cette langue qui m'accompagne en voyage. Je voyage léger, bien sûr. J'emmène mon ordinateur, des vêtements propres, un nécessaire de toilette, et quelques bouquins. Je lis dans l'avion. J'écris dans la chambre d'hôtel. Ma vie est devenue si simple. (LAFERRIÈRE, 2007, p. 87)

Dans cet extrait - où d'ailleurs réapparaît l'image de la valise -, l'écrivain commente le fait que le français soit sa langue d'écriture, même si elle n'est pas sa langue maternelle, que l'on suppose être le créole haïtien. II s'agit là d'un débat fondamental, auquel peu d'écrivains francophones contemporains échappent ${ }^{7}$. Néanmoins, Laferrière le considère comme une perte de temps, quelque chose d' " assez théorique, et même un brin ridicule ", tout en affirmant qu'il a totalement adopté la langue française. II ne convient pas, bien entendu, de juger cette position ou de revendiquer une place plus distinguée à la langue créole, par exemple, mais de reconnaître sa posture face à un nombre de questions qui animent une part importante du débat contemporain, surtout vis-à-vis des littératures francophones ou d'expression française. En outre, le traitement presque violent donné à l'accusation supposée de colonisé peut démontrer à quel degré ces questions sont importantes pour l'auteur, même s'il ne le reconnaît pas : " Mais le colonisé, je peux le dire, c'est celui qui ne se voit ni ne s'entend. II se nourrit de mensonges. Sa vie est une fiction ".

Au-delà de ces questions, un nouveau voyage se profile, cette fois-ci à New York, ville où habitait et où est décédé Windsor Laferrière, le père du narrateur. Lors de ses

7 On sait que le volume Pour une littérature-monde, organisé par Michel Le Bris et Jean Rouaud, a suivi la parution du manifeste " Pour une littérature-monde en français », dans l'édition du 15 mars 2007 du journal Le Monde. En défendant l'idée d'une production en français indépendante de la littérature nationale française, ce manifeste polémique a été signé par quarante-quatre écrivains, dont Dany Laferrière. 


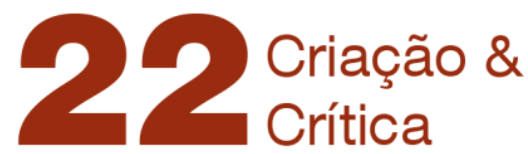

funérailles, la proximité physique du père entraîne, encore une fois, l'agencement d'un passage du poème de Césaire :

\author{
C'est la première fois \\ que je le vois de si près. \\ Je n'ai qu'allonger \\ la main pour le toucher. \\ Si je ne le fais pas \\ c'est pour respecter la distance \\ qu'il a voulu maintenir entre nous \\ de son vivant.
}

Je me rappelle ce passage dans le Cahier où Césaire réclame le corps de Toussaint Louverture arrêté par Napoléon qui devait mourir de froid durant I'hiver de 1803 au fort de Joux, en France. Les lèvres tremblantes de rage contenue du poète venu réclamer, cent cinquant ans plus tard, le corps gelé du héros de la révolte des esclaves [...]. (LAFERRIĖRE, 2009, p. 60)

Ainsi que nous l'avons déjà constaté, il existe un rapport entre la figure de Césaire et celle du père : dans cet extrait, cependant, c'est le narrateur lui-même qui s'approprie ces «lèvres tremblantes de rage contenue " pour réclamer le corps de son père. On apprend dans la suite du récit que Windsor n'était pas un « héros de la révolte des esclaves ", comme l'était Toussaint Louverture, mais quelqu'un qui avait subi l'exil à cause de ses idéaux politiques. En fait, cette contiguïté entre les destins du père et du fils (et même entre leurs noms) semble être l'une des conséquences tragiques de la montée de dictatures qui a eu lieu dans l'histoire récente d'Haïti ${ }^{8}$.

Malgré cela, on peut remarquer aussi une distance, maintenue de manière forcée par le père envers le fils, ce qui explique que les funérailles soient aussi l'occasion d'une proximité maximale entre eux. Ensuite, Dany et ses oncles vont au Chase Manhattan Bank afin de récupérer la valise que Windsor y avait déposée. Néanmoins, cette nouvelle tentative sera vouée à l'échec parce qu'ils ne connaissent pas son code secret, ce qui les empêche de l'ouvrir. Ici, ce signe de la valise devient encore plus net, dans la mesure où la prétendue transmission ne s'accomplit pas: "Cette valise m'attendait. I II a fait confiance au réflexe de son fils. / Ce qu'il ne savait pas / (tais-toi donc, on n'apprend rien à un mort) / c'est que le destin ne se transmet pas de père en fils. / Cette valise n'appartient qu'à lui. Le poids de sa vie » (2009, p. 70).

En dépit de ce désir de Windsor - qui l'avait poussé à mettre « en lieu sûr / son bien le plus précieux $»-$, cette transmission de destin de père en fils n'est pas accomplie. À propos de l'hommage rendu, tout au long de l'ouvrage, à des figures paternelles telles

8 « Mon père était un journaliste et un homme politique assez impétueux. II n'avait peur de rien. N'oublie pas que nous sommes en Haïti, au tout début du règne de François Duvalier. Mon père s'est opposé assez tôt à Duvalier [...]. Dans mon cas, il s'agit d'une symétrie tragique. François Duvalier a exilé mon père, et JeanClaude Duvalier m'exilera vingt ans plus tard. Père et fils, présidents ; père et fils, exilés. " (LAFERRIĖRE, 2000, p. 15-16). 
que celles de Césaire et de Louverture, Christiane Ndiaye, dans son article " La ruine de l'autobiographie : une mémoire littéraire sans frontières », pose les questions suivantes :

Cet hommage s'accompagne néanmoins d'un discours relativisant implicite où s'inscrit également un questionnement de l'héritage laissé par ces illustres prédécesseurs. N'ont-ils pas vécu aussi (surtout ?) des désillusions? Tout en reconnaissant leurs réalisations, ne faut-il pas se garder de marcher dans leurs pas ? Chacun n'a-t-il pas la liberté de quitter les chemins tracés par les pères et de se frayer la propre voie ? C'est du moins ce que suggère le symbolisme de la valise du père que les héritiers devront laisser à la Chase Manhattan Bank, ainsi que le rapprochement opéré par le texte entre plusieurs " pères fondateurs ». (NDIAYE, 2016, p. 45)

Tout en reconnaissant un questionnement implicite sur la transmission de l'héritage, ces suggestions peuvent nous amener à conclure que le poids d'une vie n'appartient qu'à celui qui l'a menée jusqu'au bout : en fait, la mélancolie de cette pensée accompagnera le narrateur pendant tout le reste du récit, en Haïti. Néanmoins, avant d'entreprendre ce retour au pays natal, le narrateur revient encore une fois chez lui pour terminer ses lents préparatifs de départ. Dans ce chapitre, intitulé "Dernier matin », il a envie de rencontrer son ami Rodney Saint-Éloi, même s'il ne sait pas trop pourquoi. En fait, il ne se passe presque rien en termes d'action, sinon la description quelque peu mouvementée de l'appartement où vit cet ami. II faut avoir en tête que c'est la transcription à peu près fidèle d'une personne vivante, celle du poète et éditeur Rodney Saint-Éloi, qui dirige la maison d'éditions Mémoire d'encrier, responsable de la diffusion d'un grand nombre d'écrivains haïtiens, entre autres nationalités et chez qui Laferrière avait déjà publié quelques-uns de ses ouvrages, notamment Les années 80 dans ma vieille Ford, paru cinq ans avant la publication de L'Énigme du retour.

II ne s'agit pourtant pas d'un simple hommage à l'un de ses éditeurs via son inscription dans l'œuvre elle-même, ce qui d'ailleurs pourrait sembler de mauvais goût. Effectivement, on peut lire ce chapitre comme une espèce d'approche littéraire de son Haïti natal, puisque le narrateur rappelle quelques poètes de ce pays : "Affichés sur le mur les poèmes lumineux / de Jacques Roumain, ce jeune homme qui chanta / si tristement Madrid sous la mitraille », suivi par "Une photo du poète Davertige (costume clair, melon noir et large sourire) à l'entrée. Ce sourire sous la douleur d'un dandy au repos qui me rappelle mon père " (2009, p. 71-73). Quoique très brèves, ces descriptions du célèbre poète haïtien Jacques Roumain et de Villard Denis, alias Davertige, jouent un rôle important dans l'imaginaire de l'écrivain, car elles permettent de rendre un hommage à des personnalités remarquables de la littérature haïtienne, ce qui ne va presque pas se répéter dans la suite du récit. 


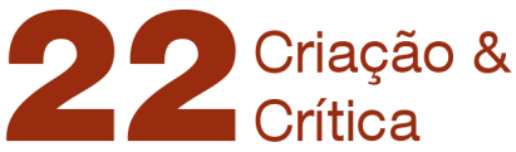

\section{Le retour ou les paradoxes de la posture}

La deuxième partie du livre ("Le retour ») suit le voyage du narrateur en Haïti, son pays natal. Quoique, en termes purement biographiques, Dany Laferrière eût déjà écrit un livre où il racontait son retour en Haïti (Pays sans chapeau, publié en 1996), ce voyage, dans L'Énigme du retour, est investi d'une charge poétique très particulière. À ce propos, écrit Anthony Soron :

Dans les deux cas, nous retrouvons la même problématique littéraire, celle de l'écriture du retour. Cependant, à douze ans d'intervalle, l'enjeu des deux romans se révèle sensiblement différent. Pays sans chapeau, à l'image de son titre étrange pour un non-Haïtien, adopte une perspective singulière tant sur le plan stylistique que narratif [...]. Nous conviendrons qu'en comparaison, l'argument de L'Énigme du retour semble plus classique. La trame de l'enquête disparaît au profit d'une recherche au sens proustien du terme [...]. Le récit du retour n'est plus structuré, comme dans le roman antérieur auquel nous nous référons, à partir de notations prises sur le vif mais plus d'évocations inspirées par le vif du sujet. (SORON, 2013, p. 150)

D’après Soron, ce qui peut distinguer les deux romans n'est pas la problématique littéraire - l'écriture du retour -, mais leurs enjeux, c'est-à-dire leurs façons singulières de traiter ce sujet si commun dans la littérature antillaise et sa diaspora. Tandis que Pays sans chapeau semble plus structuré sur le plan narratif, avec une intrigue qui relève de la question du réalisme merveilleux, l'argument de L'Énigme du retour est plus classique, même si sa trame disparaît « au profit d'une recherche au sens proustien du terme ». En effet, cette recherche du temps perdu fonctionne comme une espèce d'arrière-plan agissant au-delà de l'exil spatial : «Et l'exil du temps est plus impitoyable / que celui de l'espace. / Mon enfance / me manque plus cruellement / que mon pays » (LAFERRIĖRE, 2009, p. 73).

Cela dit, il faut résumer l'intrigue de cette deuxième partie du récit : grosso modo, le narrateur rencontre sa famille (la mère, une sœur et un neveu, qui s'appelle aussi Dany) et quelques amis de son père. II refuse de rester chez eux et s'installe à l'hôtel. En apparence, il est totalement attiré par le hasard, comme un flâneur dans son Haïti natal. En réalité, on peut s'apercevoir, au fur et à mesure que la lecture avance, qu'il essaie de s'approcher de cette figure paternelle qui reste toujours comme une énigme pour lui. Au bout du voyage, il visite Baradères, le petit village où est né Windsor Laferrière. Cependant, contrairement à ce qu'a suggéré Anthony Soron dans le titre de son article, les recherches du narrateur ne s'organisent pas « de l'obstacle à la transparence », mais se dédoublent en une série d'énigmes sans solution facile, comme dans le cas de la valise placée au Chase Manhattan Bank.

Parmi les étapes de cette intrigue en réalité très simple, le lecteur peut accompagner des mises en scène, des souvenirs, des digressions, des commentaires sur 
une multiplicité accablante de sujets. Néanmoins, en ce qui concerne le thème de la lecture, il y a des différences importantes par rapport à la première partie du récit, à commencer par le fait que le Cahier d'un retour au pays natal semble perdre de plus en plus son intérêt : "Ce matin ce n'est pas Césaire / que j'ai envie de lire / mais bien ce Lanza del Vasto / qui parvient à se satisfaire / d'un verre d'eau fraîche. / J'ai besoin d'un homme serein / et non d'un bougre en colère » (2009, p. 78).

Dans le premier chapitre ( $D$ u balcon de l'hôtel ») de la deuxième partie, on peut remarquer déjà la disparition de l'envie de lire Césaire, comme si la lecture de ce poète n'était plus appropriée face à la vue ouverte du balcon de l'hôtel. II vaut mieux donc de lire " ce Lanza del Vasto / qui parvient à se satisfaire / d'un verre d'eau fraîche », au lieu de ce «bougre en colère » qui avait permis autrefois au narrateur non pas seulement de supporter la souffrance de l'exil, mais aussi de se rapprocher du corps décédé de son père. Cela ne signifie pas qu'il n'ouvre plus jamais le volume de Césaire, mais tout se passe comme si, face au retour réel, le Cahier d'un retour au pays natal n'était plus nécessaire. Presque à la fin du livre, on peut lire ces deux passages contigus :

On nous a apporté du café. J'ai aussitôt dans la bouche le goût de Césaire. Ce Césaire qui parle de « ceux qui n'ont exploré ni les mers ni le ciel mais sans qui la terre ne serait pas la terre ». Ce sont les mêmes qui passent devant moi, dans ce petit marché qui s'anime doucement [...]

J'ai glissé dans la sacoche de mon neveu

le vieux recueil gondolé par la pluie

du Cahier d'un retour au pays natal.

C'est avant de partir qu'on en a besoin.

Pas au retour.

(LAFERRIĖRE, 2009, p. 258-259)

Cette dernière citation à Césaire permet encore une fois de mieux observer le réel : ainsi, les gens qui passent devant le narrateur, « dans ce petit marché qui s'anime doucement ", soudain deviennent les nègres héroïques et exploités dont parle le poète. Le plus important, par contre, vient dans la partie suivante, où l'exemplaire du Cahier est glissé dans la sacoche du neveu, avec cette constatation décisive, "C'est avant de partir qu'on en a besoin. / Pas au retour ». II faut bien retenir cette scène, puisqu'elle s'inscrit dans la problématique de ce que l'on a appelé le contenu de la valise et, par extension, la transmission de l'héritage. Effectivement, l'image de la sacoche, en tant que sac ouvert et servant à abriter de nouveaux objets, constitue ainsi l'exact contraire de la valise du père, qui reste close jusqu'à la fin. Néanmoins, il ne s'agit pas d'une transmission évidente et bien accomplie, parce que le neveu n'en a même pas conscience : il s'agit d'un héritage bien plus subtil, sous la forme d'un écrit, comme l'avaient été pour le narrateur la lettre de sa mère et ce « vieux recueil gondolé par la pluie / du Cahier d'un retour au pays natal ». L'expérience capable d'être transmise semble plutôt inscrite dans ces pages abîmées du volume que dans quelques éventuelles instructions pragmatiques que l'oncle Dany 
pourrait donner à son neveu Dany. La similitude des noms et la proximité des destins ainsi comme son oncle, le neveu a envie d'être écrivain et on sent que son exil s'approche de plus en plus - restent aussi comme énigme et non pas comme transmission transparente, bien résolue.

On a déjà développé partiellement la question de l'héritage, surtout dans sa relation avec l'image de la valise. En guise de conclusion provisoire, on pourrait insister sur le fait que, même si l'oncle Dany refuse d'offrir des renseignements à son neveu, la lecture reste toujours l'une des vraies possibilités de transmission d'un héritage :

II [le neveu Dany] va se chercher un verre de jus et revient à la charge. Une dernière question : est-ce mieux d'écrire à la main ou à l'ordinateur ? C'est toujours mieux de lire. D'accord, je vois que je ne vais rien tirer de vous, fait-il en prenant un Carter Brown sur la petite étagère avant de filer aux toilettes. (LAFERRIÈRE, 2009, p. 104)

Si l'acte de lecture se maintient comme la seule possibilité véritable de léguer à quelqu'un des renseignements et des expériences, il faut préciser de quelles lectures il s'agit. On a déjà constaté, en lisant d'autres textes de Laferrière, sa prédilection pour des œuvres souvent qualifiées d'universelles. En ce qui concerne le Cahier d'un retour au pays natal, on a vu que malgré son déclin tout au long du retour réel il reste l'intertexte le plus important de L'Énigme du retour. On pourrait alors s'interroger sur le(s) rapport(s) qu'entretient cet écrivain-lecteur nommé Dany Laferrière avec les figures de la littérature haïtienne qu'il rencontre in locus.

Le passage où apparaît Frankétienne est à cet égard éclairant. En réalité, il ne s'agit pas d'un chapitre sur cet important poète haïtien, auteur entre autres du roman Les affres d'un défi, mais de la narration d'une rencontre totalement aléatoire avec lui :

Malgré l'heure tardive on fait un crochet par la route de Delmas voir Frankétienne. Le médecin veut lui acheter un tableau de sa dernière manière. Frankétienne est un artiste si prolifique qu'il peut ruiner un collectionneur. II nous accueille avec un tel vacarme qu'il a dû réveiller le quartier. L'ogre dans son antre. Malgré les commentaires louangeurs du médecin, Frankétienne semble réticent à lui vendre un tableau de sa collection personnelle [...] II travaille en ce moment à un roman dont on voit le volumineux manuscrit étalé dans un énorme fouillis sur sa grande table de travail. Tout déborde chez lui. Torse nu. Appétit gargantuesque. Visage rouge comme un homard ébouillanté. Enthousiasme débridé d'un homme obsédé de littérature et de peinture. II a peint quelques milliers de tableaux et son premier grand roman Ultravocal s'est métastasé depuis quarante ans en une trentaine de volumes [...]. (LAFERRIĖRE, 2009, p. 212)

Ainsi est présenté Frankétienne, comme quelqu'un fortement marqué par le signe de l'excès, ce qui est démontré par l'usage d'expressions presque caricaturales (« L'ogre 
dans son antre ", " appétit gargantuesque », " homard ébouillanté "), aussi bien que par cette observation: "tout déborde chez lui ». Son œuvre semble ainsi dériver plutôt de l'enthousiasme de l'homme que du travail de l'artiste: les «quelques milliers de tableaux " et « son premier grand roman Ultravocal [qui] s'est métastasé depuis quarante ans en une trentaine de volumes » semblent valoir davantage par leur nombre et leur extension que par leurs aspects d'œuvre d'art. Cette impression peut être confirmée dans la suite du passage :

Me voyant perplexe devant cette tonne de papiers bariolés de signes obscurs plus proches de notes de musique que de lettres de l'alphabet (il serait capable d'inventer un vocabulaire et une grammaire afin d'écrire un livre complètement original), il me lance que sa prochaine œuvre sera un roman-opéra. C'est quoi un roman-opéra? demande alors le chauffeur qui semblait somnoler dans son coin. Frankétienne se retourne brusquement vers lui : Vous êtes le premier à oser me le demander, les autres font semblant de comprendre. Je ne peux pas l'expliquer, mais quand j'aurai fini le livre, vous verrez, en attendant, laissez-moi vous offrir un tableau. (LAFERRIÈRE, 2009, p. 212-213).

La suite de la rencontre relève encore moins de la posture de Frankétienne en tant qu'écrivain. S'il y a certes la perplexité de l'auteur-narrateur « devant cette tonne de papiers bariolés de signes obscurs », ceux-ci sont " plus proches de notes de musique que de lettres de l'alphabet ». Même la constatation de l'originalité de Frankétienne semble moins relever d'un intérêt par rapport à son œuvre que d'une espèce de caricature, d'une exagération personnelle. Ensuite, le dialogue entre Dany et le personnage de Frankétienne ne s'accomplit même pas, grâce à l'intervention du chauffeur « qui semblait somnoler dans son coin ». C'est d'ailleurs cette intervention qui permettra le don du tableau désiré par le médecin (et qui est offert non pas à celui-ci, mais au chauffeur), mettant ainsi un point final à la rencontre. Ce qui importe le plus n'est pas exactement la direction que prend la conversation, mais le fait qu'elle soit presque totalement latérale entre les deux écrivains, phénomène qui semble avoir de l'importance.

On pourrait s'interroger ainsi sur les motifs qui font en sorte que Frankétienne soit vu plutôt comme peintre que comme écrivain. D'ailleurs, on peut remarquer tout au long de la deuxième partie de L'Énigme du retour, que l'auteur-narrateur semble davantage attiré par la peinture naïve si commune en Haïti que par la littérature produite dans son pays natal : en effet, les très fréquentes allusions au Cahier donnent lieu à de multiples références aux peintres haïtiens, par exemple dans les chapitres « Un petit cimetière peint comme un tableau naïf près de Soissons-la-Montagne » et "Comment vit-on à soixantedix ans dans un musée ? ". Cette observation rejoint donc l'effort, mené par Véronique Bonnet, « d'interroger la bibliothèque cosmopolite de Laferrière tout au long des différentes étapes de sa construction » (2016, p. 265). Il est intéressant de constater qu'en interrogeant la bibliothèque de son premier roman, Comment faire l'amour avec un nègre sans se fatiguer, la critique peut conclure que « de la littérature haïtienne il ne retient que 


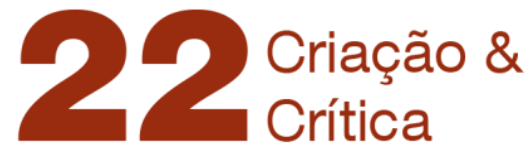

les classiques - Jacques-Stephen Alexis et Jacques Roumain [...] et non ses pairs, ceux qui occupent une place d'éventuels rivaux au sein du champ littéraire haïtiano-québecois [...]» (BONNET, 2016, p. 264).

Si dans L'Énigme du retour il n'est pas question des rivalités au niveau du champ littéraire haïtiano-québecois, puisque l'auteur était déjà très connu lors de sa rédaction, on ne peut attribuer une attitude aussi systématique au pur hasard. II s'agirait plutôt d'une manière de se mettre en rapport avec le « jeu » littéraire - cette fois-ci à l'échelle de la littérature-monde, et non pas de l'univers québecois - que l'on pourrait rapprocher de l'idée de posture, telle que développée par Jérôme Meizoz dans deux extraits du chapitre «Qu'entend-on par posture? ", au sein de son essai Postures littéraires : mises en scène modernes de l'auteur:

La « posture " est la manière singulière d'occuper une " position » dans le champ littéraire. Connaissant celle-ci, on peut décrire comment une " posture » la rejoue ou la déjoue. Qui fait imprimer un ouvrage (un disque, une gravure, etc.) impose une image de soi qui dépasse les coordonnées d'identité du citoyen [...] (MEIZOZ, 2007, p. 18)

Disons que j'entends par posture quelque chose de commun à tous les écrivains (et à tous les artistes en général), attaché à leur statut même : une façon de faire face, comme on dit, littéralement: faire (bonne ou mauvaise) figure aux avantages et désavantages de la position qu'on occupe dans le «jeu» littéraire ou plus généralement artistique. Tout écrivain manifeste une posture, consciente ou non. (MEIZOZ, 2007, p. 20)

L'un des chapitres de L'Énigme du retour le plus fortement lié à cette problématique, est celui intitulé "Éloge de la diarrhée ». À ce moment du récit, le narrateur demeure encore à Port-au-Prince, avant d'entreprendre le voyage vers Baradères, village natal de son père. Après avoir pris un « jus de fruits dans une goguette sur [le] chemin », juste pour prouver qu'il était « toujours l'enfant du pays », il court aux toilettes de l'hôtel et se met à lire « un vieux numéro d'Historia qui [lui] apprend tout sur l'ascension d'Himmler dans le Troisième Reich ». Ce fait est le déclencheur d'un flux de souvenirs qui peut avoir de l'intérêt :

Ça m'a rappelé mon adolescence du temps que ces histoires me passionnaient, ce qui désespérait ma mère qui avait une peur bleue de tout ce qui touchait de loin ou de près la politique. Pourtant elle a arrêté de trembler pour moi dès que j'avais publié mon premier article dans $L e$ Nouvelliste. C'était un long commentaire littéraire à propos de Ficus, un roman qui venait de paraître. [...] Mon article a provoqué deux réactions décisives pour ma carrière. Celle du professeur Ghislain Gouraige, l'auteur d'une monumentale Histoire de la littérature haïtienne (de l'indépendance à nos jours) qu'on étudiait encore à l'école, qui m'a félicité pour la fraîcheur de ma perception tout en me signalant une dizaine d'erreurs factuelles, 


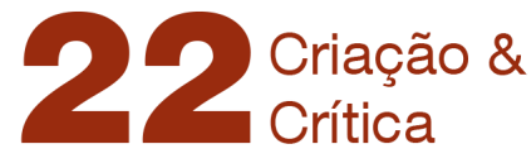

suivie dans la même journée d'une convocation aux casernes du major Valmé. C'était, selon les critères établis, la consécration. (LAFERRIÈRE, 2009, p. 176)

Assez curieux, ce souvenir organisé autour de l'article consacré à Ficus, roman de Rassoul Labuchin, se révèle intéressant d'un double point de vue, lié aux « deux réactions décisives " pour la carrière de l'écrivain Laferrière. Le premier consiste à reconnaître que lui-même, il avoue avoir eu un poste de critique littéraire en Haïti, ce qui signifie au minimum qu'il a lu ces auteurs en tant que littérature haïtienne, en accord avec l'œuvre mentionnée du professeur Gouraige. Le deuxième point de vue est associé à l'importance que prend cet épisode pour la carrière de Dany Laferrière, aussi bien que pour sa vie :

L'entrevue s'est pourtant bien déroulée et n'a pas trop débordé du cadre littéraire. À propos du roman de Rassoul Labuchin, son avis différait du mien. Pour lui, le véritable projet de Rassoul Labuchin n'était pas littéraire mais politique. Savais-je que l'auteur avait déjà séjourné à Moscou ? Qu'il était le confident de l'écrivain communiste Jacques Stephen Alexis ? Pour moi Compère général soleil de Jacques Stephen Alexis est un des plus beaux romans de la littérature haïtienne. Spontanément il me répond que sa préférence va au Romancero aux étoiles. [...] II m'a finalement félicité pour ce « style clair et lisible si peu dans la manière haïtienne ». J'ai été impressionné par l'élégance et la culture de cet homme, sans jamais perdre de vue qu'il dirigeait la chambre des tortures de Papa Doc. (LAFERRIÈRE, 2009, p. 177)

En accord avec ce «style clair et lisible » dont parlait le major Valmé, cet épisode central est raconté de manière légère et spontanée, comme si c'était l'un des effets naturels de cet « éloge de la diarrhée ». Pourtant, on peut remarquer que l'élégance et la culture de cet homme, capables d'impressionner le jeune Dany Laferrière, contrastent fortement avec son rôle de dirigeant de la chambre de tortures du dictateur Papa Doc. En réalité, les rapports entre littérature et politique, ou encore entre critique littéraire et survivance, se sont présentés comme sujet explicite de la conversation, puisque le major lui-même les a suscités, en rappelant les liaisons entre Rassoul Labuchin et « l'écrivain communiste Jacques Stephen Alexis ", l'une des plus grandes figures de la littérature haïtienne. De telle sorte que la préférence pour Compère général soleil ou pour le Romancero aux étoiles, ainsi que l'éloge du style du jeune critique, s'équilibraient de manière risquée entre les pôles de l'esthétique et de la politique. La suite des faits fera tomber cet équilibre : « J'ai tenu la chronique littéraire dans l'hebdomadaire culturel et politique Le Petit Samedi soir jusqu'à l'assassinat par les tontons macoutes de mon collègue Gasner Raymond [...]. J'ai pris l'exil tout de suite après pour Montréal » (2009, p. 177).

C'est seulement à ce moment que le lecteur du roman peut prendre conscience du fait que la raison immédiate de l'exil de Laferrière n'est pas tout simplement la politique en termes abstraits, mais les liaisons dangereuses entre celle-ci et l'esthétique, via son 


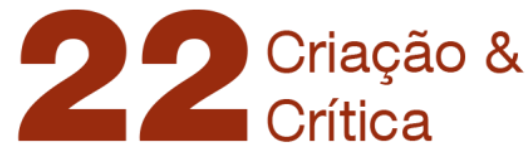

activité de critique littéraire. À l'origine de son départ, il y a ainsi à nouveau la pratique de la lecture, cette fois-ci liée explicitement aux problèmes particuliers posés par la littérature nationale haïtienne, comme peuvent le démontrer les œuvres déjà citées de Jacques Stephen Alexis. II convient donc de noter que ces questions sont à l'origine de l'exil de Dany Laferrière. Ce phénomène devient d'autant plus curieux si l'on rappelle la posture de lecteur-écrivain ou tout simplement celle de lecteur qu'il s'efforce de soutenir, soit dans ses entretiens (le cas déjà cité de J'écris comme je vis), soit dans ses textes littéraires (le cas présent de L'Énigme du retour) ou encore dans quelques récentes performances publiques, selon l'analyse proposée par Véronique Bonnet (2016).

Néanmoins, il ne convient pas, dans les limites de cet essai, de pousser ces suggestions vers des conclusions définitives. L'attitude de Dany Laferrière à l'égard de la littérature caribéenne, et notamment de la littérature haïtienne, est parfois contradictoire et paradoxale: il s'agit là encore d'une énigme non résolue. Elle pourrait être liée à la posture d'un " écrivain-monde " et à ses enjeux, à celle d'un écrivain-lecteur fréquentant des classiques universels, issu de la périphérie du système littéraire en langue française. Par ailleurs, les rapports entre vie réelle et fiction sont si proches chez Laferrière qu'il devient difficile d'analyser son œuvre sans tenir compte de sa trajectoire factuelle. En ce sens-là, il est parfaitement possible que la menace réelle représentée par le major Valmé et les tontons macoutes se soit inscrite dans la subjectivité de Dany comme un vrai trauma, capable de l'éloigner définitivement d'une approche systématique par rapport à la littérature haïtienne.

Quelle que soit la conclusion que l'on puisse en tirer, il faut retenir l'importance indéniable du rôle joué par la lecture dans l'œuvre de Laferrière et particulièrement dans L'Énigme du retour. Dès les fréquentes allusions au Cahier d'un retour au pays natal de Césaire, jusqu'aux livres et lettres laissés en héritage dans la valise de l'oncle ou dans la sacoche du neveu, la lecture reste la seule possibilité effective de la transmission de l'expérience. Finalement, pour arriver à la fin de sa recherche proustienne, après avoir visité Baradères, le narrateur se pose face à la mer et se laisse bercer « par la musique / du vieux vent caraïbe », en imaginant «la vie langoureuse d'avant Colomb» (2009, p. 278). C'est l'accomplissement de son retour :

Une main douce

sur mon front apaise la fièvre.

Je somnole entre aube et crépuscule.

Et dors le reste du temps [...]

On me vit aussi sourire

dans mon sommeil.

Comme l'enfant que je fus

du temps heureux de ma grand-mère.

Un temps enfin revenu.

C'est la fin du voyage.

(LAFERRIÈRE, 2009, p. 280) 


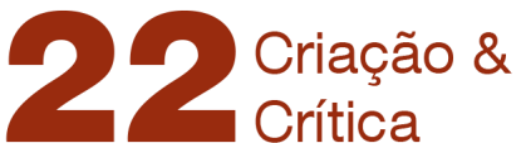

\section{Remerciements :}

Nous remercions la professeure Véronique Bonnet, dont la lecture et les suggestions ont été fondamentales pour l'écriture de ce texte.

\section{Références bibliographiques :}

ALMEIDA, L. P. de. «Posfácio ». In : CÉSAIRE, A. Diário de um retorno ao país natal. Trad. Lilian P. de Almeida. São Paulo : Edusp, 2012. p. 93-154.

BONNET, V. "Dany Laferrière : le lecteur dans sa baignoire ou l'art de bien lire ". In : PARISOT, Y. (Org.). Dany Laferrière: mythologies de l'écrivain, énergie du roman. Interculturel francophonies, Lecce, v. 3, n. 61, p. 255-279, 2016.

CÉSAIRE, A. Cahier d'un retour au pays natal. Paris : Éditions Présence Africaine, 1983.

LAFERRIĖRE, D. Journal d'un écrivain en pyjama. Paris : Bernard Grasset, 2013. . L'énigme du retour. Paris : Bernard Grasset, 2009.

« Je voyage en français ». In : Le BRIS, Michel ; ROUAUD, Jean (Dir.). Pour une littérature-monde. Paris : Gallimard, NRF, 2007. . J'écris comme je vis. Entretiens avec Bernard Magnier. Paris : Éditions La passe du vent, 2000.

MEIZOZ, J. Postures littéraires : mises en scène modernes de l'auteur. Genève : Slatkine Érudition, 2007.

NDIAYE, C. "La ruine de l'autobiographie: une mémoire littéraire sans frontières ». In : PARISOT, Y. (Org.). Dany Laferrière: mythologies de l'écrivain, énergie du roman. Interculturel francophonies, Lecce, v. 3, n. 61, p. 31-54, 2016.

SORON, A. «De l'obstacle à la transparence : L'Énigme du retour de Dany Laferrière ou la réponde limpide de l'écriture vibrante ". In : BRODZIAK, S. (Org.) et al. Haïti, enjeux d'écriture. Paris : Presses Universitaires de Vincennes, 2013.

Recebido em: 30/08/2018

Aceito em: 25/11/2018

Referência eletrônica: AMARAL, Henrique Provinzano. La Valise de L'écrivain: Variations sur le Thème de la Lecture dans L'énigme du Retour, de Dany Laferrière. Criação \& Crítica, n. 22, p., dez. $2018 . \quad$ Disponível em: $<$ http://revistas.usp.br/criacaoecritica>. Acesso em: dd mmm. aaaa. 